\title{
Small bowel bleeding in patients with left ventricular assist device: outcomes of conservative therapy versus balloon-assisted enteroscopy
}

\author{
Badr Al-Bawardya , Sarah D. Schettle ${ }^{b}$, Emmanuel Gorospea ${ }^{a}$ Louis M. Wong Kee Songa, \\ Naveen L. Pereirab, Jeffrey A. Alexandera, David H. Bruining ${ }^{a}$, Nayantara Coelho-Prabhua , Jeff L. Fidlerc, \\ William J. Mauermann ${ }^{d}$, David W. Barbarad ${ }^{d}$ Ross Dierkhising ${ }^{\mathrm{e}}$, Elizabeth Rajan ${ }^{\mathrm{a}}$
}

Mayo Clinic, Rochester, MN, USA

\section{Abstract}

\begin{abstract}
Background Small bowel bleeding (SBB) accounts for 30\% of gastrointestinal bleeding (GIB) episodes in patients with a left ventricular assist device (LVAD). The aim of this study was to determine the outcomes of conservative therapy (CT) compared to balloon-assisted enteroscopy (BAE) in the management of SBB in LVAD patients.

Methods A retrospective review was performed of a prospectively maintained LVAD database from January 2003 to July 2015. LVAD patients with SBB were classified into a BAE group or a CT group according to whether they did or did not undergo BAE.

Results Forty-two patients (22 BAE, $20 \mathrm{CT}$ ) with mean age $66 \pm 9.3$ years (79\% male) were included. The yield of BAE was $64 \%$ without reported complications. Overt re-bleeding occurred in $40 \%$ of the BAE group compared to $22 \%$ of the CT group. The BAE group had a higher mean number of GIB hospitalizations per month compared to the CT group (0.07 vs. 0.03; incidence rate ratio [IRR] 2.72, 95\% CI 1.06-6.98; $\mathrm{P}=0.04)$. There was no significant difference between the $\mathrm{BAE}$ and the CT groups in the number of packed red blood cell (pRBC) transfusions per month ( 0.42 vs. 0.18 ; IRR $2.31,95 \%$ CI $0.88-6.04 ; \mathrm{P}=0.09)$ or all-cause mortality ( $61 \%$ in the CT group and $42 \%$ in the BAE group; $\mathrm{P}=0.90$ ).

Conclusion BAE is safe in LVAD patients and has a moderate therapeutic yield. In our cohort of patients, BAE did not appear to improve re-bleeding rate, GIB-related hospitalizations, pRBC transfusions or mortality compared to CT. However, future prospective trials with larger sample sizes are needed to confirm these findings.
\end{abstract}

Keywords Small bowel bleeding, left ventricular assist device, capsule endoscopy, balloon-assisted enteroscopy

Ann Gastroenterol 2018; 31 (6): 1-6
aDivision of Gastroenterology and Hepatology (Badr Al-Bawardy, Emmanuel Gorospe, Louis M. Wong Kee Song, Jeffrey A. Alexander, David H. Bruining, Nayantara Coelho-Prabhu, Elizabeth Rajan); ${ }^{b}$ Department of Cardiology (Sarah D. Schettle, Naveen L. Pereira); 'Department of Radiology (Jeff L. Fidler); ${ }^{\mathrm{d} D e p a r t m e n t ~ o f ~}$ Anesthesiology (William J. Mauermann, David W. Barbara); ${ }^{e}$ Division of Biomedical Statistics and Informatics (Ross Dierkhising), Mayo Clinic, Rochester, Rochester, MN, USA

Conflict of Interest: None

Correspondence to: Badr Al-Bawardy, MD, Instructor in Medicine, Division of Gastroenterology \& Hepatology, Mayo Clinic, 200 First Street, S.W., Rochester, MN 55905, USA, e-mail: albawardy.badr@ mayo.edu

Received 4 April 2018; accepted 24 July 2018; published online 26 September 2018

DOI: https://doi.org/10.20524/aog.2018.0316

\section{Introduction}

Left ventricular assist device (LVAD) therapy has been shown to improve survival and quality of life in advanced heart failure compared to medical therapy [1]. Both continuous (HeartMate II, Thoratec, Pleasanton, CA; HeartWare, Framingham, MA) and pulsatile flow (HeartMate III, HeartMate XVE, Thoratec, Pleasanton, CA) LVADs have been associated with an increased risk of gastrointestinal bleeding (GIB), higher for continuous-flow devices [2-4]. Multiple factors have been postulated that may increase the risk of GIB, including low pulsatility, pharmaceutical anticoagulation and acquired von Willebrand factor disease [5-8].

The reported prevalence of GIB in LVAD patients ranges from 16-23\% [9-11]. In a recent meta-analysis, small bowel bleeding (SBB) accounted for $15 \%$ of GIB events in LVAD 
patients. However, the rate of SBB has been noted to be as high as $31 \%$ [12]. Angioectasia is the most common culprit lesion detected [11]. The optimal management strategy of SBB in LVAD patients is not clearly defined and has not been widely studied. Small case series have demonstrated the safety of balloon-assisted enteroscopy (BAE) [13,14]. However, there are limited data on the long-term outcomes of BAE in LVAD patients.

The aim of this study was to determine the long-term outcomes of conservative therapy (CT) compared to BAE in the management of SBB in LVAD patients.

\section{Patients and methods}

We conducted a retrospective review of a prospectively maintained LVAD database from January 2003 to July 2015. The study was approved by the Institutional Review Board. Patients with GIB after LVAD implantation were identified. SBB was defined as GIB in patients with normal upper endoscopy and colonoscopy. The study included patients who had overt or occult SBB and underwent BAE, or capsule endoscopy (CE) or computed tomography enterography (CTE). Overt GIB was defined as melena or hematochezia and occult GIB was defined as iron deficiency anemia and positive fecal occult blood.

Patients who met the inclusion criteria were classified into two groups. The CT group included patients who had SBB treated with either oral or parenteral iron replacement and/ or blood transfusions. These patients had a CE and/or CTE performed but did not undergo BAE. The BAE group included all LVAD patients who had SBB treated with either oral or parenteral iron replacement and/or blood transfusions and underwent BAE. Patients in both the $\mathrm{CT}$ and BAE groups who received medical therapy for GIB, such as octreotide or danazol, at the discretion of the primary clinician were identified. The decision for patients to undergo BAE was made by the primary clinician. Balloon-assisted enteroscopy was performed with either single-balloon (SIF-Q180, Olympus Corp., Center Valley, Pennsylvania, USA) or double-balloon (EN-450T5, Fujinon, Inc., Saitama, Japan) enteroscopes. The decision to use a single versus a double balloon and an antegrade or retrograde approach was guided by the presumed location of the culprit lesion, detected by small bowel diagnostic imaging when available. Actively bleeding and any potential bleeding sites (such as non-bleeding angioectasia) were treated endoscopically during BAE. Re-bleeding was defined as recurrence of overt bleeding after initial BAE or after small bowel diagnostic imaging.

Baseline comparisons between the $\mathrm{CT}$ and $\mathrm{BAE}$ groups were made using Wilcoxon rank-sum tests for continuous variables and Pearson's Chi-square tests for discrete variables. Death after the initial small bowel evaluation was compared between groups using Kaplan-Meier methodology and the log-rank test. The periods before and after the initial small bowel evaluation for the number of GIB hospitalizations and packed red blood cell (pRBC) transfusions per month were compared using Poisson regression with generalized estimating equations to account for the potential correlation between periods. The number of GIB hospitalizations and pRBC transfusions per month after the initial small bowel evaluation were compared between the BAE and CT groups using Poisson regression. Incidence rate ratios (IRRs) with 95\% confidence intervals (CI) were computed from the Poisson models to compare incidence rates. The incidence of having a BAE after the initial small bowel evaluation in the BAE group was estimated using Kaplan-Meier methodology with adjustment for the competing risk of death.

\section{Results}

A total of 322 LVAD patients were reviewed. From this cohort, 97 patients (30\%) had GIB post LVAD placement. Forty-two patients met the inclusion criteria (Fig. 1).

\section{Baseline characteristics}

The mean age $( \pm$ standard deviation $[\mathrm{SD}])$ was $66 \pm 9.3$ years and 33 patients (79\%) were male. A total of 22 patients underwent BAE and were classified in the BAE group while 20 patients underwent conservative management and were classified in the CT group. There was no statistically significant difference between the groups in terms of age, sex, etiology of heart failure, use of LVAD as destination therapy and chronic kidney disease requiring hemodialysis (Table 1 ). LVAD as destination therapy was employed in $71 \%$ of the study cohort. The proportion of patients who had HeartMate II implantation was $80 \%$ in the CT group and $91 \%$ in the BAE group $(\mathrm{P}=0.31)$. The median follow-up time after small bowel evaluation was 38 months and 28 months in the CT and BAE groups, respectively.

Overt GIB was observed in $65 \%$ of the CT group and $59 \%$ of the BAE group $(\mathrm{P}=0.69)$. Among the 26 overt GIB patients, the most common presentation was melena $(85 \%$ in the BAE vs. $69 \%$ in the CT group; $\mathrm{P}=0.35$ ). The mean time from LVAD implantation to first GIB was $241 \pm 369$ days and $154 \pm 216$ days $(\mathrm{P}=0.91)$ in the $\mathrm{CT}$ and $\mathrm{BAE}$ groups, respectively. There was no significant difference between the groups in the history of GIB pre-LVAD implantation (Table 1).

At the time of small bowel evaluation, the mean hemoglobin $(9.2 \pm 1.0 \mathrm{~g} / \mathrm{dL}$ vs. $9.4 \pm 1.1 \mathrm{~g} / \mathrm{dL} ; \mathrm{P}=0.60)$ and international normalized ratio (INR) $(1.5 \pm 0.8$ vs. $1.2 \pm 0.3$; $\mathrm{P}=0.08$ ) levels were similar between the $\mathrm{CT}$ and $\mathrm{BAE}$ groups, respectively. The mean platelet count was $260 \pm 122$ in the CT group and $171 \pm 54(\mathrm{x} 1000 / \mathrm{uL})$ in the $\mathrm{BAE}$ group $(\mathrm{P}=0.004)$.

The BAE group was less likely to be on aspirin (68\% vs. $95 \% ; \mathrm{P}=0.03)$ or warfarin $(82 \%$ vs. $100 \% ; \mathrm{P}=0.05)$. There was no significant difference between the $\mathrm{CT}$ and BAE groups in the use of clopidogrel ( $10 \%$ vs. $14 \% ; \mathrm{P}=0.72)$. Among the 40 patients taking antithrombotic medication, the rate of de-escalation was similar in the CT and BAE groups (65\% vs. $75 \%$; $\mathrm{P}=0.49$ ). A total of 8 patients $(36 \%)$ in the BAE group and 1 patient $(5 \%)$ in the CT group were on medical therapy for GIB. In the BAE group 7 patients were on danazol and 1 patient was on octreotide, while one patient in the CT group 


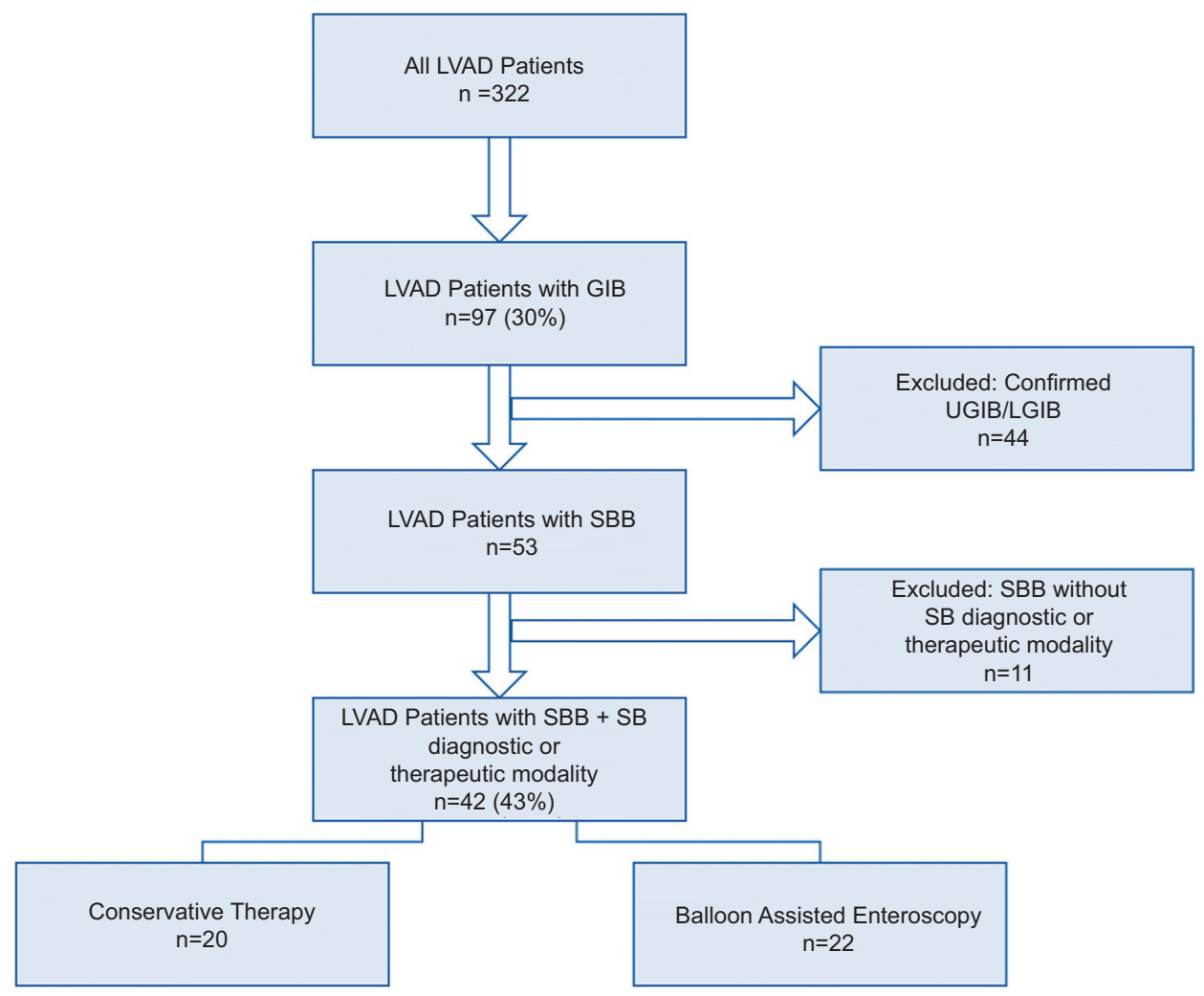

Figure 1 Study inclusion flow diagram

LVAD, left ventricular assist device; GIB, gastrointestinal bleeding; UGIB, upper gastrointestinal bleeding; LGIB, lower gastrointestinal bleeding; SBB, small bowel bleeding; SB, small bowel

Table 1 Baseline characteristics of LVAD patients with small bowel bleeding

\begin{tabular}{|c|c|c|c|c|}
\hline Characteristics & $\mathrm{CT}(\mathrm{N}=20)$ & BAE $(\mathrm{N}=22)$ & Total $(\mathrm{N}=42)$ & P-value \\
\hline Age, mean (SD) & $63.7(10.2)$ & $68.6(7.9)$ & $66.3(9.3)$ & 0.09 \\
\hline Male, n (\%) & $15(75.0)$ & $18(81.8)$ & $33(78.6)$ & 0.59 \\
\hline Etiology of heart failure, $\mathrm{n}(\%)$ & & & & 0.60 \\
\hline Ischemic & $12(60.0)$ & $11(50.0)$ & $23(54.8)$ & \\
\hline Dilated & $7(35.0)$ & $8(36.4)$ & $15(35.7)$ & \\
\hline Other & $1(5.0)$ & $3(13.6)$ & $4(9.5)$ & \\
\hline Type of LVAD, n (\%) & & & & 0.31 \\
\hline HeartMate II & $16(80.0)$ & $20(90.9)$ & $36(85.7)$ & \\
\hline HeartWare & $4(20.0)$ & $2(9.1)$ & $6(14.3)$ & \\
\hline LVAD as destination therapy, $\mathrm{n}(\%)$ & $15(75.0)$ & $15(68.2)$ & $30(71.4)$ & 0.63 \\
\hline $\begin{array}{l}\text { History of GIB prior to } \\
\text { LVAD, } n(\%)\end{array}$ & $3(15.0)$ & $6(27.3)$ & $9(21.4)$ & 0.33 \\
\hline Hemodialysis, n (\%) & $1(5.0)$ & $3(13.6)$ & $4(9.5)$ & 0.34 \\
\hline
\end{tabular}

was on danazol. These therapies were initiated after SBB was confirmed with CE or CTE.

CE was positive in $67 \%(n=12)$ and $93 \%(n=14)$ of the CT and $\mathrm{BAE}$ groups, respectively $(\mathrm{P}=0.12)$ with visible blood and angioectasia being the most common findings. In the BAE group, one patient had a false negative $\mathrm{CE}$ with angioectasia found on BAE. There were no reported adverse events relating to the performance of CE in LVAD patients. A total of 6 patients had both CE and CTE. Small bowel evaluation modalities and findings are summarized in Table 2. 
Table 2 Small bowel evaluation findings

\begin{tabular}{|c|c|c|c|c|}
\hline Evaluation modality & $\mathrm{CT}(\mathrm{N}=20)$ & BAE $(\mathrm{N}=22)$ & Total $(\mathrm{N}=42)$ & P-value \\
\hline Capsule endoscopy, n (\%) & $18(90)$ & $15(68)$ & $33(79)$ & 0.09 \\
\hline Active bleeding & $2(11)$ & $5(33.3)$ & $8(24.2)$ & 0.05 \\
\hline Angioectasia & $4(22.2)$ & $4(26.7)$ & $8(24.2)$ & 0.77 \\
\hline Altered heme & $5(27.8)$ & $5(33.3)$ & $12(36.4)$ & 0.69 \\
\hline Ulceration & $1(5.6)$ & $0(0.0)$ & $4(12.1)$ & 0.38 \\
\hline Gastric retention & $1(5.6)$ & $0(0.0)$ & $1(3.0)$ & 0.35 \\
\hline Negative & $5(27.8)$ & $1(6.7)$ & $6(18.2)$ & 0.12 \\
\hline CTE, n (\%) & $5(25)$ & $5(22.7)$ & $10(24)$ & 0.86 \\
\hline Angioectasia & $0(0.0)$ & $2(40)$ & $2(20)$ & \\
\hline Negative & $5(100)$ & $3(60)$ & $8(80)$ & \\
\hline
\end{tabular}

More than one evaluation modality and finding per patient is possible.

$B A E$, balloon-assisted enteroscopy; CT, conservative therapy; CTE, computed tomography enterography; LVAD, left ventricular assist device

\section{BAE findings and outcomes}

The diagnostic and therapeutic yields of BAE were both $64 \%$. The most common finding was angioectasia (64\%). Thermal therapy was utilized in the majority of patients (64\%). There were no adverse events related to BAE procedures. The likelihood of having a repeat BAE was $35 \%$ at 1 year and $43 \%$ at 3 years after the initial BAE. Balloon-assisted enteroscopy procedure details and findings are summarized in Table 3.

In the BAE group, the number of GIB-related hospitalizations per month decreased by $77 \%$ (IRR $0.23,95 \% \mathrm{CI}$ 0.11-0.50; $\mathrm{P}<0.001)$ after the initial BAE. The number of $\mathrm{pRBC}$ transfusions per month decreased by $82 \%$ (IRR $0.18,95 \% \mathrm{CI}$ 0.06-0.60; $\mathrm{P}=0.005)$ after the initial BAE.

\section{CT vs. BAE outcomes}

Overt re-bleeding occurred in $22 \%$ (median follow-up time of 38 months) in the CT group compared to $40 \%$ (median follow-up time of 28 months) in the BAE group. The BAE group had a significantly higher number of GIB-related hospitalizations per month compared to the CT group ( 0.07 vs. 0.03; IRR 2.72, 95\%CI 1.06-6.98; $\mathrm{P}=0.04$ ). The number of pRBC transfusions per month was 0.42 in the BAE group compared to 0.18 in the CT group (IRR 2.31, 95\%CI 0.88-6.04; $\mathrm{P}=0.09$ ). All-cause mortality was $61 \%$ in the CT group and $42 \%$ in the BAE group 36 months after the initial small bowel evaluation ( $\mathrm{P}=0.90)$ (Fig. 2). There were no GIB-related deaths.

\section{Discussion}

In our cohort of LVAD patients with SBB, patients who underwent BAE did not have superior objective clinical outcomes in terms of GIB-related hospitalizations, number of pRBC transfusions, overt re-bleeding, or all-cause or GIB-related mortality compared to patients who underwent
Table 3 BAE findings

\begin{tabular}{lc}
\hline Procedure details/findings & BAE $\mathrm{n}=22$ \\
\hline Inpatient, $\mathrm{n}(\%)$ & $20(91)$ \\
Route of BAE, $\mathrm{n}(\%)$ & $12(55)$ \\
$\quad$ Antegrade & $10(45)$ \\
Retrograde & \\
Findings, $\mathrm{n}(\%)$ & $9(41)$ \\
Actively bleeding angioectasia & $5(23)$ \\
Non-bleeding angioectasia & $6(27)$ \\
Negative & $2(9)$ \\
Unsuccessful exam & $14(64)$ \\
Treatment modality, $\mathrm{n}(\%)$ & $9(64)$ \\
Thermal (APC or bipolar) & $1(7)$ \\
Mechanical (hemoclip) & $4(29)$ \\
Combination (mechanical + thermal) &
\end{tabular}

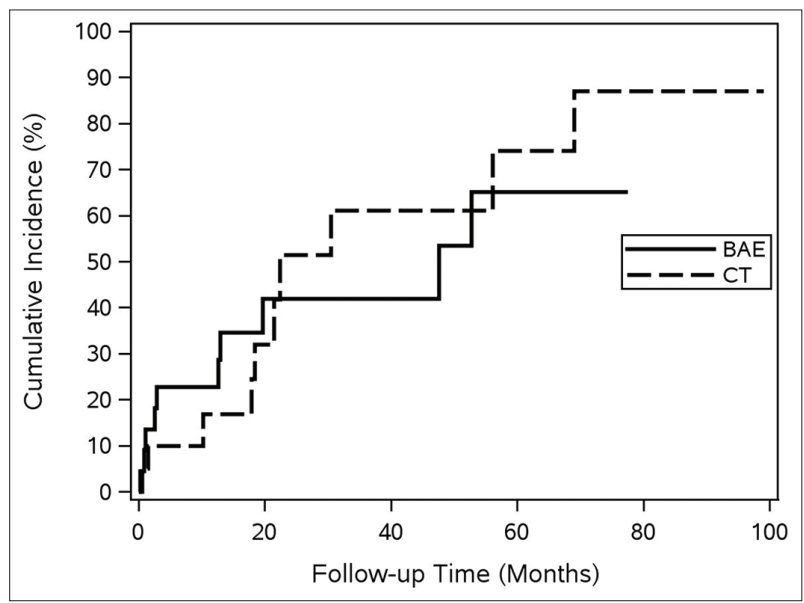

Figure 2 Incidence of all-cause mortality in the BAE group and CT group $B A E$, balloon-assisted enteroscopy; $C T$, conservative therapy 
CT. We noted, however, that after the initial BAE, the number of GIB-related hospitalizations and $\mathrm{pRBC}$ transfusions per month improved in the BAE group. BAE was safe, without documented adverse events.

GIB is a known complication of LVAD therapy. The overall rate of GIB in our cohort was 30\%, comparable to other published series $[3,5,15]$. Multiple pathophysiologic mechanisms have been proposed to account for the greater incidence of GIB in LVAD patients. Acquired von Willebrand disease is one key mechanism that has been described $[5,16,17]$. It has been well reported that continuous-flow devices are associated with a greater risk of GIB compared to pulsatile LVADs [2,3]. Increased intraluminal pressure, coupled with lower pulse pressure, leading to transient intestinal hypoperfusion appears to increase the risk of developing angioectasia in patients with continuous-flow LVADs [18].

Small bowel angioectasia treated by BAE carries a substantial risk of re-bleeding. In non-LVAD patients, multiple studies have shown the risk of re-bleeding after therapeutic BAE to be as high as $40-46 \%[19,20]$. Our study showed similar results, with a re-bleeding rate of $40 \%$ following the initial BAE.

The safety and periprocedural management of LVAD patients undergoing upper endoscopy and colonoscopy has been well studied [21]. However, data regarding the safety and utility of BAE in this patient population are sparse. Edwards et al reported on the safety and findings of BAE in a small group of 10 LVAD patients with suspected SBB [13]. The diagnostic yield in that cohort was $69 \%$, comparable to our diagnostic yield of $64 \%$ with no reported adverse events. The diagnostic yield of antegrade BAE is generally higher than that of retrograde BAE [22]. Kwong et al reviewed 28 cases of deep enteroscopy performed in LVAD patients and found no adverse events [23].

Our study appears to be the first to compare CT to BAE in LVAD patients with GIB; furthermore, although the sample size is small, it still represents the largest experience to date. This study is limited by its single-center nature and the inherent limitations of a retrospective study. Our study groups were similar in characteristics relevant to GIB, including age, INR, type of LVAD, and proportion of patients on destination therapy. Notably, while the BAE patients were less likely to be on aspirin and warfarin, they had more GIB-related hospitalizations and pRBC transfusions. Although the difference did not reach statistical significance, the CT group had a numerically higher all-cause mortality rate of $61 \%$, compared to $42 \%$ in the BAE group. There was also a higher proportion of patients in the BAE group with a positive $\mathrm{CE}$ and more were on medical therapy (danazol and octreotide). Even though a history of GIB, active bleeding and chronic kidney disease requiring hemodialysis showed no statistically significant difference between the two groups, all were numerically more frequent in the BAE group, which may indicate more refractory or severe GIB compared to the CT group. Since these cumulative differences may potentially play a role in outcomes, additional studies with larger patient populations are needed to further support our findings. Another limitation of the study is physician referral bias, as patients referred for BAE may have been less stable; however, objective parameters such as

\section{Summary Box}

\section{What is already known:}

- The rate of small bowel bleeding (SBB) in left ventricular assist device (LVAD) patients can approach $31 \%$

- Long-term outcomes of SBB in LVAD patients have not been widely studied

- The optimal management strategy for SBB (balloon-assisted enteroscopy [BAE] vs. conservative therapy $[\mathrm{CT}]$ ) has not been clearly defined in LVAD patients

\section{What the new findings are:}

- BAE is safe in LVAD patients with SBB

- BAE has a moderate therapeutic yield in LVAD patients with SBB (64\%)

- Compared to CT, BAE did not appear to improve long-term clinical outcomes, such as re-bleeding rate, hospitalizations, transfusion requirements, or mortality

mean hemoglobin, renal function, overt GIB and the use of antiplatelet/anticoagulation medication were not significantly different from those in the CT group.

In conclusion, BAE is safe in LVAD patients and has a moderate therapeutic yield. In our study population, performing BAE did not appear to reduce GIB-related hospitalizations, re-bleeding, pRBC transfusions or all-cause mortality compared to conservative management. However, future prospective trials with larger cohorts of patients are needed to confirm these findings.

\section{References}

1. Rose EA, Gelijns AC, Moskowitz AJ, et al; Randomized Evaluation of Mechanical Assistance for the Treatment of Congestive Heart Failure (REMATCH) Study Group. Long-term use of a left ventricular assist device for end-stage heart failure. $N$ Engl J Med 2001;345:1435-1443.

2. Crow S, John R, Boyle A, et al. Gastrointestinal bleeding rates in recipients of nonpulsatile and pulsatile left ventricular assist devices. J Thorac Cardiovasc Surg 2009;137:208-215.

3. Stern DR, Kazam J, Edwards P, et al. Increased incidence of gastrointestinal bleeding following implantation of the heartmate II LVAD. J Card Surg 2010;25:352-356.

4. Islam S, Cevik C, Madonna R, et al. Left ventricular assist devices and gastrointestinal bleeding: a narrative review of case reports and case series. Clin Cardiol 2013;36:190-200.

5. Uriel N, Pak SW, Jorde UP, et al. Acquired von Willebrand syndrome after continuous-flow mechanical device support contributes to a high prevalence of bleeding during long-term support and at the time of transplantation. J Am Coll Cardiol 2010;56:1207-1213. 
6. Klovaite J, Gustafsson F, Mortensen SA, Sander K, Nielsen LB. Severely impaired von Willebrand factor-dependent platelet aggregation in patients with a continuous-flow left ventricular assist device (HeartMate II). J Am Coll Cardiol 2009;53:2162-2167.

7. Meyer AL, Malehsa D, Budde U, Bara C, Haverich A, Strueber M. Acquired von Willebrand syndrome in patients with a centrifugal or axial continuous flow left ventricular assist device. JACC Heart Fail 2014;2:141-145.

8. Wever-Pinzon O, Selzman CH, Drakos SG, et al. Pulsatility and the risk of nonsurgical bleeding in patients supported with the continuous-flow left ventricular assist device HeartMate II. Circ 2013;6:517-526.

9. Singh G, Albeldawi M, Kalra SS, Mehta PP, Lopez R, Vargo JJ. Features of patients with gastrointestinal bleeding after implantation of ventricular assist devices. Clin Gastroenterol Hepatol 2015;13:107-114.e101.

10. Shrode CW, Draper KV, Huang RJ, et al. Significantly higher rates of gastrointestinal bleeding and thromboembolic events with left ventricular assist devices. Clin Gastroenterol Hepatol 2014;12:1461-1467.

11. Draper KV, Huang RJ, Gerson LB. GI bleeding in patients with continuous-flow left ventricular assist devices: a systematic review and meta-analysis. Gastrointest Endosc 2014;80:435-446.e1.

12. Morgan JA, Paone G, Nemeh HW, et al. Gastrointestinal bleeding with the HeartMate II left ventricular assist device. J Heart Lung Transplant 2012;31:715-718.

13. Edwards AL, Mönkemüller K, Pamboukian SV, George JF, Wilcox CM, Peter S. Utility of double-balloon enteroscopy in patients with left ventricular assist devices and obscure overt gastrointestinal bleeding. Endoscopy 2014;46:986-991.

14. Decker GA, Miller ED, Pasha SF, Harrison ME, Leighton JA. Deep enteroscopy in patients with left ventricular assist devices: practical and technical considerations. Endoscopy 2010;42(Suppl 2): E194.

15. Stulak JM, Lee D, Haft JW, et al. Gastrointestinal bleeding and subsequent risk of thromboembolic events during support with a left ventricular assist device. J Heart Lung Transplant 2014;33:60-64.

16. Geisen U, Heilmann C, Beyersdorf F, et al. Non-surgical bleeding in patients with ventricular assist devices could be explained by acquired von Willebrand disease. Eur J Cardiothorac Surg 2008;33:679-684.

17. Crow S, Chen D, Milano C, et al. Acquired von Willebrand syndrome in continuous-flow ventricular assist device recipients. Ann Thorac Surg 2010;90:1263-1269.

18. Suarez J, Patel CB, Felker GM, et al. Mechanisms of bleeding and approach to patients with axial-flow left ventricular assist devices. Circ 2011;4:779-784.

19. Samaha E, Rahmi G, Landi B, et al. Long-term outcome of patients treated with double balloon enteroscopy for small bowel vascular lesions. Am J Gastroenterol 2012;107:240-246.

20. May A, Friesing-Sosnik T, Manner H, Pohl J, Ell C. Long-term outcome after argon plasma coagulation of small-bowel lesions using double-balloon enteroscopy in patients with mid-gastrointestinal bleeding. Endoscopy 2011;43:759-765.

21. Barbara DW, Olsen DA, Pulido JN, et al. Periprocedural management of 172 gastrointestinal endoscopies in patients with left ventricular assist devices. ASAIO J 2015;61:670-675.

22. Sanaka MR, Navaneethan U, Kosuru B, Yerneni H, Lopez R, Vargo JJ. Antegrade is more effective than retrograde enteroscopy for evaluation and management of suspected small-bowel disease. Clin Gastroenterol Hepatol 2012;10:910-916.

23. Kwong WT, Pearlman M, Kalmaz D. Safety of deep enteroscopy and capsule endoscopy in LVAD patients: case report and literature review. Gastroenterol Res 2015;8:309-312. 